\title{
Multidimensional Hausdorff operators and commutators on Herz-type spaces
}

\author{
Amjad Hussain ${ }^{1,2^{*}}$ and Guilian Gao
}

\section{"Correspondence:}

ahabbasi123@yahoo.com

'Department of Mathematics,

Zhejiang University, Hangzhou,

310027, P.R. China

${ }^{2}$ Department of Mathematics,

HITEC University, Taxila Cannt, Taxila, Pakistan

\begin{abstract}
In this paper, we give necessary and sufficient conditions for the boundedness of the $n$-dimensional Hausdorff operators on Herz-type spaces. In addition, the sufficient condition for the boundedness of commutators generated by Lipschitz functions and the fractional Hausdorff operators on Morrey-Herz space is also provided.
\end{abstract}

MSC: 26D15; 42B35; 42B99

Keywords: Hausdorff operators; Herz-type spaces; Lipschitz functions; commutators

\section{Introduction}

Recall that for a locally integrable function $\Phi$ on $(0, \infty)$, the one-dimensional Hausdorff operator is defined by

$$
h_{\Phi} f(x)=\int_{0}^{\infty} \frac{\Phi(t)}{t} f\left(\frac{x}{t}\right) d t .
$$

The boundedness of this operator on the real Hardy space $H^{1}(R)$ was proved in [1]. Subsequently, the problem of boundedness of $h_{\Phi}$ in $H^{p}, 0<p<1$ was considered in $[2,3]$ and [4]. In [5], the same operator was studied on product of Hardy spaces. Due to its close relation with the summability of the classical Fourier series, it was natural to study $h_{\Phi}$ in high-dimensional space $R^{n}$. With such an objective, Chen et al. [6] considered three extensions of the one-dimensional Hausdorff operator in $R^{n}$. One of them is the operator

$$
H_{\Phi} f(x)=\int_{R^{n}} \frac{\Phi(y)}{|y|^{n}} f\left(\frac{x}{|y|}\right) d y .
$$

The second multidimensional extension of the Hausdorff operator provided in [6] is the following operator:

$$
\widetilde{H}_{\Phi, \Omega} f(x)=\int_{R^{n}} \frac{\Phi(x /|y|)}{|y|^{n}} \Omega\left(y^{\prime}\right) f(y) d y
$$

where $\Phi$ is a radial function defined on $R^{+}$, and $\Omega\left(y^{\prime}\right)$ is an integrable function defined on the unit sphere $S^{n-1}$. Here and in what follows, we denote $\widetilde{H}_{\Phi, 1}=\widetilde{H}_{\Phi}$. In [6], the authors discussed the boundedness of these operators on various function spaces and found that they have better performance on Herz-type Hardy spaces $H \dot{K}_{q}^{\alpha, p}$ than their performance on the Hardy spaces $H^{p}$ when $0<p<1$.

@2013 Hussain and Gao; licensee Springer. This is an Open Access article distributed under the terms of the Creative Commons Attribution License (http://creativecommons.org/licenses/by/2.0), which permits unrestricted use, distribution, and reproduction in any medium, provided the original work is properly cited. 
Recently, Lin and Sun [4] defined the $n$-dimensional fractional Hausdorff operator initially on the Schwartz class $\mathrm{S}$ by

$$
H_{\Phi, \gamma}=\int_{R^{n}} \frac{\Phi(|x| /|y|)}{|y|^{n-\gamma}} f(y) d y, \quad 0 \leq \gamma<n
$$

and obtained $H^{p}\left(R^{n}\right) \rightarrow L^{q}\left(R^{n}\right)$ and $L^{p}\left(|x|^{\alpha} d x\right) \rightarrow L^{q}\left(|x|^{\alpha} d x\right)$ boundedness for $H_{\Phi, \gamma}$. Furthermore, it is easy to show that the $n$-dimensional fractional Hardy operator

$$
H_{\gamma} f(x)=\frac{1}{|x|^{n-\gamma}} \int_{|y|<|x|} f(y) d y
$$

and its adjoint operator

$$
H_{\gamma}^{*} f(x)=\int_{|y| \geq|x|} \frac{f(y)}{|y|^{n-\gamma}} d y
$$

are special cases of $H_{\Phi, \gamma}$ if one chooses $\Phi(t)=\Phi_{1}(t)=t^{-n+\gamma} \chi_{(1, \infty)}(t)$ and $\Phi(t)=\Phi_{2}(t)=$ $\chi_{(0,1]}(t)$, respectively.

In recent years, the interest in obtaining sharp bounds for integral operators has grown rapidly, mainly because of their appearance in various branches of pure and applied sciences. In [7], Xaio obtained the sharp bounds for the Hardy Littlewood averaging operator on Lebesgue and BMO spaces. Later on the problem was extended to $p$-adic fields in [8] and [9]. In [10] and [11], Fu with different co-author have considered the same problem for $m$-linear $p$-adic Hardy and classical Hardy operators, respectively.

As the development of linear as well as multilinear integral operators, their commutators have been well studied. A well-known theorem by Coifman et al. [12] states that the commutator $[b, T]$ defined by

$$
[b, T](f)(x)=b(x) T(f)(x)-T(b f)(x)
$$

where $T$ is a Calderón-Zygmund singular integral operator, is bounded on $L^{p}\left(R^{n}\right)$, $1<p<\infty$, if and only if $b \in B M O\left(R^{n}\right)$. One can find a vast literature devoted to the study of the boundedness properties for such commutators. More recently, Gao and Jia [13] defined the commutator of the high-dimensional Hausdorff operator as

$$
\widetilde{H}_{\Phi, b} f(x)=\int_{R^{n}} \frac{\Phi(x /|y|)}{|y|^{n}}(b(x)-b(y)) f(y) d y
$$

and studied it on Lebesgue and Herz-type spaces.

Motivated by the work cited above, in this paper, we obtain some sharp bounds for $H_{\Phi}$ on Herz-type spaces. Furthermore, we give a sufficient condition for the boundedness of commutators generated by the Lipschitz functions $b$ and the $n$-dimensional fractional Hausdorff operators $H_{\Phi, \gamma}$, defined by

$$
H_{\Phi, \gamma}^{b} f(x)=\int_{R^{n}} \frac{\Phi(|x| /|y|)}{|y|^{n-\gamma}}(b(x)-b(y)) f(y) d y,
$$

on Morrey-Herz space. Following [14], our method is direct and straightforward. In addition, the problem of boundedness of commutators of $n$-dimensional fractional Hardy 
operators [15] is also achieved as a special case of our results. Before going into the detailed proof of these results, let us first recall some definitions. For any $k \in Z$, we set $B_{k}=\left\{x \in R^{n}:|x| \leq 2^{k}\right\}, C_{k}=B_{k} \backslash B_{k-1}$.

Definition 1.1 ([16]) Let $\alpha \in R, 0<p \leq \infty, 0<q<\infty$. The homogeneous Herz space $\dot{K}_{q}^{\alpha, p}\left(R^{n}\right)$ is defined by

$$
\dot{K}_{q}^{\alpha, p}\left(R^{n}\right)=\left\{f \in L_{\mathrm{loc}}^{q}\left(R^{n} \backslash\{0\}\right):\|f\|_{\dot{K}_{q}^{\alpha, p}\left(R^{n}\right)}<\infty\right\},
$$

where

$$
\|f\|_{\dot{K}_{q}^{\alpha, p}\left(R^{n}\right)}=\left(\sum_{k=-\infty}^{\infty} 2^{k \alpha p}\left\|f \chi_{C_{k}}\right\|_{L^{q}\left(R^{n}\right)}^{p}\right)^{1 / p},
$$

with the usual modification made when $p=\infty$.

Remark 1.2 $\dot{K}_{q}^{\alpha, p}\left(R^{n}\right)$ is the generalization of $L^{q}\left(R^{n},|x|^{\alpha}\right)$, the Lebesgue space with power weights. Also, it is easy to see that $\dot{K}_{q}^{0, q}\left(R^{n}\right)=L^{q}\left(R^{n}\right)$ and $\dot{K}_{q}^{\alpha / q, q}\left(R^{n}\right)=L^{q}\left(R^{n},|x|^{\alpha}\right)$.

Definition 1.3 Let $\alpha \in R, 0<p \leq \infty, 0<q<\infty$ and $\lambda \geq 0$. The homogeneous MorreyHerz space $M \dot{K}_{p, q}^{\alpha, \lambda}\left(R^{n}\right)$ is defined by

$$
M \dot{K}_{p, q}^{\alpha, \lambda}\left(R^{n}\right)=\left\{f \in L_{\mathrm{loc}}^{q}\left(R^{n} \backslash\{0\}\right):\|f\|_{M \dot{K}_{p, q}^{\alpha, \lambda}\left(R^{n}\right)}<\infty\right\},
$$

where

$$
\|f\|_{M \dot{K}_{p, q}^{\alpha, \lambda}\left(R^{n}\right)}=\sup _{k_{0} \in Z} 2^{-k_{0} \lambda}\left(\sum_{k=-\infty}^{k_{0}} 2^{k \alpha p}\left\|f \chi_{C_{k}}\right\|_{L^{q}\left(R^{n}\right)}^{p}\right)^{1 / p},
$$

with the usual modification made when $p=\infty$.

In [17] the Morrey space $M_{q}^{\lambda}\left(R^{n}\right)$ is defined by

$$
M_{q}^{\lambda}\left(R^{n}\right)=\left\{f \in L_{\mathrm{loc}}^{q}\left(R^{n}\right): \sup _{\lambda>0, x \in R^{n}} \frac{1}{r^{\lambda}} \int_{|x-y|<r}|f(y)|^{q} d y<\infty\right\} .
$$

Obviously, $M \dot{K}_{p, q}^{\alpha, 0}\left(R^{n}\right)=\dot{K}_{q}^{\alpha, p}\left(R^{n}\right)$ and $M_{q}^{\lambda}\left(R^{n}\right) \subset M \dot{K}_{q, q}^{0, \lambda}\left(R^{n}\right)$.

Definition 1.4 ([18]) Let $0<\beta<1$. The Lipschitz space $\dot{\Lambda}_{\beta}\left(R^{n}\right)$ is defined by

$$
\|f\|_{\dot{\Lambda}_{\beta}\left(R^{n}\right)}=\sup _{x, h \in R^{n}} \frac{|f(x+h)-f(x)|}{|h|^{\beta}}<\infty .
$$

In the next section we will obtain some sharp bounds for $H_{\Phi}$. Finally, the Lipschitz estimates for the commutators $H_{\Phi, \gamma}^{b}$ will be studied in the last section. 


\section{Sharp bounds for $H_{\Phi}$}

The main result of this section is as follows:

Theorem 2.1 Let $\alpha \in R, \lambda \geq 0,1<p, q<\infty$. If $\Phi$ is a non-negative valued function and

$$
A_{1}=\int_{R^{n}} \frac{\Phi(y)}{|y|^{n}}|y|^{\alpha+\frac{n}{q}-\lambda} d y<\infty
$$

then $H_{\Phi}$ is a bounded operator on $M \dot{K}_{p, q}^{\alpha, \lambda}\left(R^{n}\right)$.

Conversely, suppose that $H_{\Phi}$ is a bounded operator on $M \dot{K}_{p, q}^{\alpha, \lambda}\left(R^{n}\right)$. If $\lambda=0$, or if $\lambda>$ $\max \{0, \alpha\}$, then $A_{1}<\infty$. In addition, the operator $H_{\Phi}$ satisfies the following operator norm:

$$
\left\|H_{\Phi}\right\|_{M \dot{K}_{p, q}^{\alpha, \lambda}\left(R^{n}\right) \rightarrow M \dot{K}_{p, q}^{\alpha, \lambda}\left(R^{n}\right)}=A_{1} .
$$

Proof By definition and using Minkowski's inequality

$$
\begin{aligned}
\left\|H_{\Phi} f\right\|_{M \dot{K}_{p, q}^{\alpha, \lambda}\left(R^{n)}\right.} & =\sup _{k_{0} \in Z} 2^{-k_{0} \lambda}\left\{\sum_{k=-\infty}^{k_{0}} 2^{k \alpha p}\left\|\left(H_{\Phi} f\right) \chi_{C_{k}}\right\|_{L^{q}\left(R^{n}\right)}^{p}\right\}^{\frac{1}{p}} \\
& =\sup _{k_{0} \in Z} 2^{-k_{0} \lambda}\left\{\sum_{k=-\infty}^{k_{0}} 2^{k \alpha p}\left(\int_{C_{k}}\left|\int_{R^{n}} \frac{\Phi(y)}{|y|^{n}} f\left(\frac{x}{|y|}\right) d y\right|^{q} d x\right)^{\frac{p}{q}}\right\}^{\frac{1}{p}} \\
& \leq \sup _{k_{0} \in Z} 2^{-k_{0} \lambda}\left\{\sum_{k=-\infty}^{k_{0}} 2^{k \alpha p}\left(\sum_{j=-\infty}^{\infty} \int_{C_{j}} \frac{\Phi(y)}{|y|^{n}}\left\|f\left(\frac{\cdot}{|y|}\right)\right\|_{L^{q}\left(C_{k}\right)} d y\right)^{p}\right\}^{\frac{1}{p}} .
\end{aligned}
$$

Now, it is easy to see that for $y \in C_{j}[6]$

$$
\left\|f\left(\frac{\cdot}{|y|}\right)\right\|_{L^{q}\left(C_{k}\right)}=|y|^{\frac{n}{q}}\left\|f \chi_{C_{k-j}}\right\|_{L^{q}\left(R^{n}\right)} .
$$

Therefore, by Minkowski's inequality, we get

$$
\begin{aligned}
\left\|H_{\Phi} f\right\|_{M \dot{K}_{p, q}^{\alpha, \lambda}\left(R^{n}\right)} & \leq \sum_{j=-\infty}^{\infty} \int_{C_{j}} \frac{\Phi(y)}{|y|^{n}}|y|^{\frac{n}{q}} \sup _{k_{0} \in Z} 2^{-k_{0} \lambda}\left\{\sum_{k=-\infty}^{k_{0}} 2^{k \alpha p}\left\|f \chi_{C_{k-j}}\right\|_{L^{q}\left(R^{n}\right)}^{p}\right\}^{\frac{1}{p}} d y \\
& \leq\|f\|_{M \dot{K}_{p, q}^{\alpha, \lambda}\left(R^{n}\right)} \sum_{j=-\infty}^{\infty} \int_{C_{j}} \frac{\Phi(y)}{|y|^{n}}|y|^{\frac{n}{q}} 2^{-j(\lambda-\alpha)} d y \\
& \leq\|f\|_{M \dot{K}_{p, q}^{\alpha, \lambda}\left(R^{n}\right)} \int_{R^{n}} \frac{\Phi(y)}{|y|^{n}}|y|^{\alpha+\frac{n}{q}-\lambda} d y .
\end{aligned}
$$

Hence, we conclude that

$$
\left\|H_{\Phi}\right\|_{M \dot{K}_{p, q}^{\alpha, \lambda}\left(R^{n}\right) \rightarrow M \dot{K}_{p, q}^{\alpha, \lambda}\left(R^{n}\right)} \leq A_{1}
$$

Conversely, suppose that $H_{\Phi}$ is bounded on $M \dot{K}_{p, q}^{\alpha, \lambda}\left(R^{n}\right)$. Then we consider the following two cases.

Case I: $\lambda>0$. 
In this case, we choose $f_{0} \in L_{\text {loc }}^{q}\left(R^{n} \backslash\{0\}\right)$, such that

$$
f_{0}(x)=|x|^{-\alpha-\frac{n}{q}+\lambda}
$$

An easy computation shows that

$$
\left\|f_{0} \chi_{C_{k}}\right\|_{L^{q}\left(R^{n}\right)}=2^{k(\lambda-\alpha)}\left[\frac{\left(1-2^{q(\alpha-\lambda)}\right)\left|S^{n-1}\right|}{\lambda-\alpha}\right]^{\frac{1}{q}}
$$

where $\left|S^{n-1}\right|$ denotes the volume of unit sphere $S^{n-1}$. Now, by definition

$$
\begin{aligned}
\left\|f_{0}\right\|_{M \dot{K}_{p, q}^{\alpha, \lambda}\left(R^{n}\right)} & =\sup _{k_{0} \in Z} 2^{-k_{0} \lambda}\left\{\sum_{k=-\infty}^{k_{0}} 2^{k \alpha p}\left\|f_{0} \chi_{C_{k}}\right\|_{L^{q}\left(R^{n}\right)}^{p}\right\}^{\frac{1}{p}} \\
& =\left[\frac{\left(1-2^{q(\alpha-\lambda)}\right)\left|S^{n-1}\right|}{\lambda-\alpha}\right]^{\frac{1}{q}} \sup _{k_{0} \in Z} 2^{-k_{0} \lambda}\left\{\sum_{k=-\infty}^{k_{0}} 2^{k \lambda p}\right\}^{\frac{1}{p}} \\
& =\left[\frac{\left(1-2^{q(\alpha-\lambda)}\right)\left|S^{n-1}\right|}{\lambda-\alpha}\right]^{\frac{1}{q}} \frac{2^{\lambda}}{\left(2^{\lambda p}-1\right)^{\frac{1}{p}}}<\infty .
\end{aligned}
$$

On the other hand, it is easy to check that

$$
H_{\Phi} f_{0}(x)=f_{0}(x) \int_{R^{n}} \frac{\Phi(y)}{|y|^{n}}|y|^{\alpha+\frac{n}{q}-\lambda} d y
$$

Under the assumption that $H_{\Phi}$ is bounded on $M \dot{K}_{p, q}^{\alpha, \lambda}\left(R^{n}\right)$, we get

$$
\int_{R^{n}} \frac{\Phi(y)}{|y|^{n}}|y|^{\alpha+\frac{n}{q}-\lambda} d y \leq\left\|H_{\Phi}\right\|_{M \dot{K}_{p, q}^{\alpha, \lambda}\left(R^{n}\right) \rightarrow M \dot{K}_{p, q}^{\alpha, \lambda}\left(R^{n}\right)}<\infty .
$$

Furthermore, combing (2.2) with (2.1), we immediately obtain

$$
\left\|H_{\Phi}\right\|_{M \dot{K}_{p, q}^{\alpha, \lambda}\left(R^{n}\right) \rightarrow M \dot{K}_{p, q}^{\alpha, \lambda}\left(R^{n}\right)}=\int_{R^{n}} \frac{\Phi(y)}{|y|^{n}}|y|^{\alpha+\frac{n}{q}-\lambda} d y .
$$

Case II: $\lambda=0$.

In this case, we have $M \dot{K}_{p, q}^{\alpha, \lambda}\left(R^{n}\right)=\dot{K}_{q}^{\alpha, p}\left(R^{n}\right)$. To prove the converse relation we take the sequence of function $\left\{f_{m}\right\}(m \geq 0)$ as follows:

$$
f_{m}(x)= \begin{cases}0 & \text { if }|x|<1 \\ |x|^{-\alpha-\frac{n}{q}-2^{-m}} & \text { if }|x| \geq 1\end{cases}
$$

Obviously for $k<0$, we have $f_{m} \chi_{C_{k}}=0$. Hence, for $k \geq 0$, we obtain

$$
\begin{aligned}
\left\|f_{m} \chi_{C_{k}}\right\|_{L^{q\left(R^{n}\right)}}^{q} & =\int_{C_{k}}|x|^{-\alpha-\frac{n}{q}-2^{-m}} d x \\
& =\frac{\left(2^{q\left(\alpha+2^{-m}\right)}-1\right)}{q\left(\alpha+2^{-m}\right)}|S|^{n-1} 2^{-k q\left(\alpha+2^{-m}\right)} .
\end{aligned}
$$


Hussain and Gao Journal of Inequalities and Applications 2013, 2013:594

Page 6 of 12

http://www.journalofinequalitiesandapplications.com/content/2013/1/594

Therefore,

$$
\begin{aligned}
\left\|f_{m}\right\|_{\dot{K}_{q}^{\alpha, p}\left(R^{n}\right)} & =\left\{\sum_{k=-\infty}^{\infty} 2^{k \alpha p}\left\|f_{m} \chi_{C_{k}}\right\|_{L^{q}\left(R^{n}\right)}^{p}\right\}^{\frac{1}{p}} \\
& =\left[\frac{\left(2^{q\left(\alpha+2^{-m}\right)}-1\right)}{q\left(\alpha+2^{-m}\right)}|S|^{n-1}\right]^{\frac{1}{q}}\left\{\sum_{k=0}^{\infty} 2^{k \alpha p} 2^{-k p\left(\alpha+2^{-m}\right)}\right\}^{\frac{1}{p}} \\
& =\left[\frac{\left(2^{q\left(\alpha+2^{-m}\right)}-1\right)}{q\left(\alpha+2^{-m}\right)}|S|^{n-1}\right]^{\frac{1}{q}} \frac{2^{\frac{1}{2^{m}}}}{\left(2^{\frac{p}{2^{m}}}-1\right)^{\frac{1}{p}}}<\infty .
\end{aligned}
$$

On the other hand, we write

$$
H_{\Phi} f_{m}(x)= \begin{cases}0 & \text { if }|x|<1 \\ |x|^{-\alpha-\frac{n}{q}-2^{-m}} \int_{|y| \leq|x|} \frac{\Phi(y)}{|y|^{n}}|y|^{\alpha+\frac{n}{q}+2^{-m}} d y & \text { if }|x| \geq 1\end{cases}
$$

This implies that $\left(H_{\Phi} f_{m}\right) \chi_{C_{k}}=0$ for $k<0$. Thus for $k \geq 0$, we get

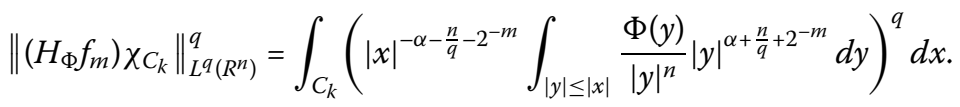

Therefore, for any $m \leq k$, we have

$$
\begin{aligned}
\left\|\left(H_{\Phi} f_{m}\right) \chi_{C_{k}}\right\|_{L^{q}\left(R^{n}\right)} \geq & \left(\int_{C_{k}}|x|^{-\alpha q-n-2^{-m} q} d x\right)^{\frac{1}{q}} \int_{|y| \leq 2^{m-1}} \frac{\Phi(y)}{|y|^{n}}|y|^{\alpha+\frac{n}{q}+2^{-m}} d y \\
= & 2^{-k\left(\alpha+2^{-m}\right)}\left[\frac{\left(2^{q\left(\alpha+2^{-m}\right)}-1\right)}{q\left(\alpha+2^{-m}\right)}|S|^{n-1}\right]^{\frac{1}{q}} \\
& \times \int_{|y| \leq 2^{m-1}} \frac{\Phi(y)}{|y|^{n}}|y|^{\alpha+\frac{n}{q}+2^{-m}} d y .
\end{aligned}
$$

Now, it is easy to show that

$$
\begin{aligned}
\left\|H_{\Phi} f_{m}\right\|_{\dot{K}_{q}^{\alpha, p}\left(R^{n}\right)} \geq & {\left[\frac{\left(2^{q\left(\alpha+2^{-m}\right)}-1\right)}{q\left(\alpha+2^{-m}\right)}|S|^{n-1}\right]^{\frac{1}{q}}\left\{\sum_{k=m}^{\infty} 2^{-\frac{k p}{2^{m}}}\right\}^{\frac{1}{p}} } \\
& \times \int_{|y| \leq 2^{m-1}} \frac{\Phi(y)}{|y|^{n}}|y|^{\alpha+\frac{n}{q}+2^{-m}} d y \\
= & {\left[\frac{\left(2^{q\left(\alpha+2^{-m}\right)}-1\right)}{q\left(\alpha+2^{-m}\right)}|S|^{n-1}\right]^{\frac{1}{q}}\left\{\sum_{k=0}^{\infty} 2^{-\frac{k p}{2^{m}}}\right\}^{\frac{1}{p}} } \\
& \times 2^{-\frac{m}{2^{m}}} \int_{|y| \leq 2^{m-1}} \frac{\Phi(y)}{|y|^{n}|y|^{\alpha+\frac{n}{q}+2^{-m}} d y} \\
= & || f_{m} \|_{\dot{K}_{q}^{\alpha, p}\left(R^{n}\right)} 2^{-\frac{m}{2^{m}}} \int_{|y| \leq 2^{m-1}} \frac{\Phi(y)}{|y|^{n}}|y|^{\alpha+\frac{n}{q}+2^{-m}} d y .
\end{aligned}
$$


Consequently,

$$
\left\|H_{\Phi}\right\|_{\dot{K}_{q}^{\alpha, p}\left(R^{n}\right) \rightarrow \dot{K}_{q}^{\alpha, p}\left(R^{n}\right)} \geq 2^{-\frac{m}{2^{m}}} \int_{|y| \leq 2^{m-1}} \frac{\Phi(y)}{|y|^{n}}|y|^{\alpha+\frac{n}{q}+2^{-m}} d y .
$$

Finally, we let $m \rightarrow+\infty$ to obtain

$$
\left\|H_{\Phi}\right\|_{\dot{K}_{q}^{\alpha, p}\left(R^{n}\right) \rightarrow \dot{K}_{q}^{\alpha, p}\left(R^{n}\right)} \geq \int_{R^{n}} \frac{\Phi(y)}{|y|^{n}}|y|^{\alpha+\frac{n}{q}} d y .
$$

In view of (2.3) with (2.1), we get

$$
\left\|H_{\Phi}\right\|_{\dot{K}_{q}^{\alpha, p}\left(R^{n}\right) \rightarrow \dot{K}_{q}^{\alpha, p}\left(R^{n}\right)}=\int_{R^{n}} \frac{\Phi(y)}{|y|^{n}}|y|^{\alpha+\frac{n}{q}} d y .
$$

Thus, we finish the proof of Theorem 2.1.

\section{Lipschitz estimates for $\boldsymbol{n}$-dimensional fractional Hausdorff operator}

In this section, we will prove that the commutator generated by Lipschitz function $b$ and the fractional Hausdorff operator $H_{\Phi, \gamma}$ is bounded on the Morrey-Herz space. Similar estimates for high-dimensional fractional Hardy operators are also obtained as a special case of the following theorem.

Theorem 3.1 Let $b \in \dot{\Lambda}_{\beta}\left(R^{n}\right), 0<\beta<1<q_{2}<q_{1}<\infty, 0<p<\infty, \lambda>0, \mu=\alpha+\beta+\gamma+$ $\frac{n}{q_{2}}-\frac{n}{q_{1}}$. If

$$
A_{2}=\int_{0}^{\infty} \frac{|\Phi(t)|}{t} t^{\alpha+\frac{n}{q_{2}}-\lambda} \max \left\{1, t^{\beta}\right\} d t<\infty,
$$

then $H_{\Phi, \gamma}^{b}$ is bounded from $M \dot{K}_{p, q_{1}}^{\mu, \lambda}\left(R^{n}\right)$ to $M \dot{K}_{p, q_{2}}^{\alpha, \lambda}\left(R^{n}\right)$ and satisfies the following inequality:

$$
\left\|H_{\Phi, \gamma}^{b} f\right\|_{M \dot{K}_{p, q_{2}}^{\alpha, \lambda}\left(R^{n}\right)} \leq C A_{2}\|b\|_{\dot{\Lambda}_{\beta}\left(R^{n}\right)}\|f\|_{M \dot{K}_{p, q_{1}}^{\mu, \lambda}\left(R^{n}\right)} .
$$

In proving Theorem 3.1, we need the following lemmas.

Lemma 3.2 For $1<p<\infty$, we have

$$
\left\|\left(H_{\Phi, \gamma} f\right) \chi_{C_{k}}\right\|_{L^{p}\left(R^{n}\right)} \leq 2^{k \gamma}\left|S^{n-1}\right| \int_{0}^{\infty} \frac{|\Phi(t)|}{t^{1+\gamma}} t^{\frac{n}{p}} \mid f \chi_{t^{-1} C_{k}} \|_{L^{p}\left(R^{n}\right)} d t .
$$

Proof The lemma can be proved in a way similar to Theorem 3.1 in [6].

Lemma 3.3 ([18]) For any $x, y \in R^{n}$, if $f \in \dot{\Lambda}_{\beta}\left(R^{n}\right), 0<\beta<1$, then $|f(x)-f(y)| \leq \mid x-$ $\left.y\right|^{\beta} \mid f \|_{\dot{\Lambda}_{\beta}\left(R^{n}\right)}$. Furthermore, for any cube $Q \subset R^{n}, \sup _{x \in Q}\left|f(x)-f_{Q}\right| \leq C|Q|^{\frac{\beta}{n}}\|f\|_{\dot{\Lambda}_{\beta}\left(R^{n}\right)}$, where $f_{Q}=\frac{1}{|Q|} \int_{Q} f$.

Lemma 3.4 ([18]) Let $f \in \dot{\Lambda}_{\beta}\left(R^{n}\right), 0<\beta<1, Q$ and $Q^{*}$ are cubes in $R^{n}$. If $Q^{*} \subset Q$, then

$$
\left|f_{Q^{*}}-f_{Q}\right| \leq C|Q|^{\frac{\beta}{n}}\|f\|_{\dot{\Lambda}_{\beta}\left(R^{n}\right)} .
$$


Proof of Theorem 3.1 Notice that

$$
\begin{aligned}
\left\|\left(H_{\Phi, \gamma}^{b} f\right) \chi_{C_{k}}\right\|_{L^{q_{2}\left(R^{n}\right)}}= & \left\|\left(\int_{R^{n}} \frac{\Phi(|x| /|y|)}{|y|^{n-\gamma}}(b(x)-b(y)) f(y) d y\right) \chi_{C_{k}}\right\|_{L^{q_{2}\left(R^{n}\right)}} \\
\leq & \left\|\left(\int_{R^{n}} \frac{\Phi(|x| /|y|)}{|y|^{n-\gamma}}\left(b(x)-b_{B_{k}}\right) f(y) d y\right) \chi_{C_{k}}\right\|_{L^{q_{2}\left(R^{n}\right)}} \\
& +\left\|\left(\int_{R^{n}} \frac{\Phi(|x| /|y|)}{|y|^{n-\gamma}}\left(b(y)-b_{B_{k}}\right) f(y) d y\right) \chi_{C_{k}}\right\|_{L^{q_{2}\left(R^{n}\right)}} \\
= & I+J .
\end{aligned}
$$

Let $\frac{1}{r}=\frac{1}{q_{2}}-\frac{1}{q_{1}}$. Then by Hölder's inequality, Lemma 3.2, and Lemma 3.3, we have

$$
\begin{aligned}
I & \leq\left(\int_{C_{k}}\left|b(x)-b_{B_{k}}\right|^{r} d x\right)^{\frac{1}{r}}\left(\int_{C_{k}}\left|\int_{R^{n}} \frac{\Phi(|x| /|y|)}{|y|^{n-\gamma}} f(y) d y\right|^{q_{1}} d x\right)^{\frac{1}{q_{1}}} \\
& \leq C\left|B_{k}\right|^{\frac{\beta}{n}+\frac{1}{r}}\|b\|_{\dot{\Lambda}_{\beta}\left(R^{n}\right)}\left\|\left(H_{\Phi, \gamma} f\right) \chi_{C_{k}}\right\|_{L^{q_{1}\left(R^{n}\right)}} \\
& \leq C 2^{k\left(\beta+\gamma+\frac{n}{r}\right)}\|b\|_{\dot{\Lambda}_{\beta}\left(R^{n}\right)} \int_{0}^{\infty} \frac{|\Phi(t)|}{t^{1+\gamma}} t^{\frac{n}{q_{1}}}\left\|f \chi_{t^{-1} C_{k}}\right\|_{L^{q_{1}\left(R^{n}\right)}} d t .
\end{aligned}
$$

Now, using polar coordinates, Minkowski's inequality and Hölder's inequality, we approximate $J$ as

$$
\begin{aligned}
J & =\left\|\left(\int_{0}^{\infty} \int_{S^{n-1}} \frac{\Phi(|x| / r)}{r^{1-\gamma}}\left(b\left(r y^{\prime}\right)-b_{B_{k}}\right) f\left(r y^{\prime}\right) d \sigma\left(y^{\prime}\right) d r\right) \chi_{C_{k}}\right\|_{L^{q_{2}\left(R^{n}\right)}} \\
& =\left\|\left(\int_{0}^{\infty} \int_{S^{n-1}} \frac{\Phi(t)}{t}\left(|x| t^{-1}\right)^{\gamma}\left(b\left(|x| t^{-1} y^{\prime}\right)-b_{B_{k}}\right) f\left(|x| t^{-1} y^{\prime}\right) d \sigma\left(y^{\prime}\right) d t\right) \chi_{C_{k}}\right\|_{L^{q_{2}\left(R^{n}\right)}} \\
& \leq 2^{k \gamma} \int_{0}^{\infty} \frac{|\Phi(t)|}{t^{1+\gamma}}\left\|\left(\int_{S^{n-1}}\left(b\left(|x| t^{-1} y^{\prime}\right)-b_{B_{k}}\right) f\left(|x| t^{-1} y^{\prime}\right) d \sigma\left(y^{\prime}\right)\right) \chi_{C_{k}}\right\|_{L^{q_{2}\left(R^{n}\right)}} d t \\
& \leq 2^{k \gamma}\left|S^{n-1}\right|^{\frac{1}{q_{2}^{\prime}}} \int_{0}^{\infty} \frac{|\Phi(t)|}{t^{1+\gamma}}\left(\int_{C_{k}} \int_{S^{n-1}}\left|\left(b\left(|x| t^{-1} y^{\prime}\right)-b_{B_{k}}\right) f\left(|x| t^{-1} y^{\prime}\right)\right|^{q_{2}} d \sigma\left(y^{\prime}\right) d x\right)^{\frac{1}{q_{2}}} d t .
\end{aligned}
$$

Again by means of polar decomposition and change of the variables, we obtain

$$
\begin{aligned}
J \leq & 2^{k \gamma}\left|S^{n-1}\right| \int_{0}^{\infty} \frac{|\Phi(t)|}{t^{1+\gamma}}\left(\int_{2^{k-1}}^{2^{k}} s^{n-1} \int_{S^{n-1}}\left|\left(b\left(s t^{-1} y^{\prime}\right)-b_{B_{k}}\right) f\left(s t^{-1} y^{\prime}\right)\right|^{q_{2}} d \sigma\left(y^{\prime}\right) d s\right)^{\frac{1}{q_{2}}} d t \\
= & C 2^{k \gamma} \int_{0}^{\infty} \frac{|\Phi(t)|}{t^{1+\gamma}} t^{\frac{n}{q_{2}}}\left(\int_{t^{-1} C_{k}}\left|\left(b(y)-b_{B_{k}}\right) f(y)\right|^{q_{2}} d y\right)^{\frac{1}{q_{2}}} d t \\
\leq & C 2^{k \gamma} \int_{0}^{\infty} \frac{|\Phi(t)|}{t^{1+\gamma}} t^{\frac{n}{q_{2}}}\left(\int_{t^{-1} C_{k}}\left|\left(b(y)-b_{t^{-1} B_{k}}\right) f(y)\right|^{q_{2}} d y\right)^{\frac{1}{q_{2}}} d t \\
& +C 2^{k \gamma} \int_{0}^{\infty} \frac{|\Phi(t)|}{t^{1+\gamma}} t^{\frac{n}{q_{2}}}\left(\int_{t^{-1} C_{k}}\left|\left(b_{B_{k}}-b_{t^{-1} B_{k}}\right) f(y)\right|^{q_{2}} d y\right)^{\frac{1}{q_{2}}} d t \\
= & J_{1}+J_{2} .
\end{aligned}
$$


For $J_{1}$, using Hölder's inequality and Lemma 3.3, we have

$$
\begin{aligned}
J_{1} & \leq C 2^{k \gamma} \int_{0}^{\infty} \frac{|\Phi(t)|}{t^{1+\gamma}} t^{\frac{n}{q_{2}}}\left(\int_{t^{-1} C_{k}}\left|b(x)-b_{t^{-1} B_{k}}\right|^{r} d x\right)^{\frac{1}{r}}\left(\int_{t^{-1} C_{k}}|f(y)|^{q_{1}} d y\right)^{\frac{1}{q_{1}}} d t \\
& \leq C 2^{k\left(\beta+\gamma+\frac{n}{r}\right)}\|b\|_{\dot{\Lambda}_{\beta}\left(R^{n}\right)} \int_{0}^{\infty} \frac{|\Phi(t)|}{t^{1+\gamma}} t^{\frac{n}{q_{1}}}\left\|f \chi_{t^{-1} C_{k}}\right\|_{L^{q_{1}\left(R^{n}\right)}} t^{-\beta} d t .
\end{aligned}
$$

Observe that if $t<1$, then $B_{k} \subset t^{-1} B_{k}$, while the reverse is true for $t>1$. Hence, by Lemma 3.4, we obtain

$$
\begin{aligned}
J_{2}= & C 2^{k \gamma} \int_{0}^{\infty} \frac{|\Phi(t)|}{t^{1+\gamma}} t^{\frac{n}{q_{2}}}\left(\int_{t^{-1} C_{k}}|f(y)|^{q_{2}} d y\right)^{\frac{1}{q_{2}}}\left|b_{B_{k}}-b_{t^{-1} B_{k}}\right| d t \\
\leq & C 2^{k \gamma}\left|B_{k}\right|^{\frac{1}{r}} \int_{0}^{\infty} \frac{|\Phi(t)|}{t^{1+\gamma}} t^{\frac{n}{q_{1}}}\left(\int_{t^{-1} C_{k}}|f(y)|^{q_{1}} d y\right)^{\frac{1}{q_{1}}}\left|b_{B_{k}}-b_{t^{-1} B_{k}}\right| d t \\
\leq & C 2^{k\left(\beta+\gamma+\frac{n}{r}\right)}\|b\|_{\dot{\Lambda}_{\beta}\left(R^{n}\right)} \int_{0}^{1} \frac{|\Phi(t)|}{t^{1+\gamma} t^{\frac{n}{q_{1}}}}\left\|f \chi_{t^{-1} C_{k}}\right\|_{L^{q_{1}\left(R^{n}\right)} t^{-\beta}} d t \\
& +C 2^{k\left(\beta+\gamma+\frac{n}{r}\right)}\|b\|_{\dot{\Lambda}_{\beta}\left(R^{n}\right)} \int_{1}^{\infty} \frac{|\Phi(t)|}{t^{1+\gamma}} t^{\frac{n}{q_{1}}}\left\|f \chi_{t^{-1} C_{k}}\right\|_{L^{q_{1}\left(R^{n}\right)}} d t \\
\leq & C 2^{k\left(\beta+\gamma+\frac{n}{r}\right)}\|b\|_{\dot{\Lambda}_{\beta}\left(R^{n}\right)} \int_{0}^{\infty} \frac{|\Phi(t)|}{t^{1+\gamma}} t^{\frac{n}{q_{1}}}\left\|f \chi_{t^{-1} C_{k}}\right\|_{L^{q_{1}\left(R^{n}\right)} \max \left\{1, t^{-\beta}\right\} d t .}
\end{aligned}
$$

Note that for $t>1,0<\beta<1$, we have $0<t^{-\beta}<1$. Therefore, by combining the estimates for $I, J_{1}$, and $J_{2}$, we get

$$
\begin{aligned}
\left\|\left(H_{\Phi, \gamma}^{b} f\right) \chi_{C_{k}}\right\|_{L^{q_{2}\left(R^{n}\right)}} \leq & C 2^{k\left(\beta+\gamma+\frac{n}{r}\right)}\|b\|_{\dot{\Lambda}_{\beta}\left(R^{n}\right)} \\
& \times \int_{0}^{\infty} \frac{|\Phi(t)|}{t^{1+\gamma}} t^{\frac{n}{q_{1}}}\left\|f \chi_{t^{-1} C_{k}}\right\|_{L^{q_{1}\left(R^{n}\right)}} \max \left\{1, t^{-\beta}\right\} d t .
\end{aligned}
$$

Following [19], we let $m \in Z$ such that $m-1<-\log _{2} t \leq m$, then $t^{-1} C_{k}$ is contained in two adjacent annuli $C_{k+m}$ and $C_{k+m-1}$. Therefore,

$$
\begin{aligned}
\left\|\left(H_{\Phi, \gamma}^{b} f\right) \chi_{C_{k}}\right\|_{L^{q_{2}\left(R^{n}\right)}} \leq & C 2^{k\left(\beta+\gamma+\frac{n}{r}\right)}\|b\|_{\dot{\Lambda}_{\beta}\left(R^{n}\right)} \\
& \times \int_{0}^{\infty} \frac{|\Phi(t)|}{t^{1+\gamma}} t^{\frac{n}{q_{1}}} \sum_{i=0}^{1}\left\|f \chi_{C_{k+m-i}}\right\|_{L^{q_{1}\left(R^{n}\right)}} \max \left\{1, t^{-\beta}\right\} d t .
\end{aligned}
$$

Hereafter, we use the notation $\widetilde{\Phi}(t)=\frac{|\Phi(t)|}{t^{1+\gamma}} t^{\frac{n}{q_{1}}} \max \left\{1, t^{-\beta}\right\}$ for simplicity. Then for $0<p<1$, we get

$$
\begin{aligned}
& \left\|H_{\Phi, \gamma}^{b} f\right\|_{M \dot{K}_{p, q_{2}}^{\alpha, \lambda}\left(R^{n}\right)} \\
& \leq C\|b\|_{\dot{\Lambda}_{\beta}\left(R^{n}\right)} \sup _{k_{0} \in Z} 2^{-k_{0} \lambda}\left\{\sum_{k=-\infty}^{k_{0}} 2^{k \mu p}\left(\int_{0}^{\infty} \widetilde{\Phi}(t)\left\|f \chi_{C_{k+m}}\right\|_{L^{q_{1}\left(R^{n}\right)}} d t\right)^{p}\right\}^{\frac{1}{p}} \\
& \quad+C\|b\|_{\dot{\Lambda}_{\beta}\left(R^{n}\right)} \sup _{k_{0} \in Z} 2^{-k_{0} \lambda}\left\{\sum_{k=-\infty}^{k_{0}} 2^{k \mu p}\left(\int_{0}^{\infty} \widetilde{\Phi}(t)\left\|f \chi_{C_{k+m-1}}\right\|_{L^{q_{1}\left(R^{n}\right)}} d t\right)^{p}\right\}^{\frac{1}{p}} \\
& =K_{1}+K_{2} .
\end{aligned}
$$


Here, we approximate $K_{1}$ as

$$
\begin{aligned}
K_{1} \leq & \sup _{k_{0} \in Z} 2^{-k_{0} \lambda}\left\{\sum_{k=-\infty}^{k_{0}} 2^{k \mu p}\right. \\
& \left.\times\left(\int_{0}^{\infty} \widetilde{\Phi}(t) 2^{-(k+m) \lambda}\left(\sum_{i=-\infty}^{k+m} 2^{i \mu p}\left\|f \chi_{C_{i}}\right\|_{L^{q_{1}\left(R^{n}\right)}}^{p}\right)^{\frac{1}{p}} 2^{(k+m)(\lambda-\mu)} d t\right)^{p}\right\}^{\frac{1}{p}} C\|b\|_{\dot{\Lambda}_{\beta}\left(R^{n}\right)} \\
\leq & C\|b\|_{\dot{\Lambda}_{\beta}\left(R^{n}\right)}\|f\|_{M \dot{K}_{p, q_{1}}^{\mu, \lambda}\left(R^{n}\right)} \sup _{k_{0} \in Z} 2^{-k_{0} \lambda}\left\{\sum_{k=-\infty}^{k_{0}} 2^{k \lambda p}\left(\int_{0}^{\infty} \widetilde{\Phi}(t) 2^{m(\lambda-\mu)} d t\right)^{p}\right\}^{\frac{1}{p}} \\
\leq & C\|b\|_{\dot{\Lambda}_{\beta}\left(R^{n}\right)}\|f\|_{M \dot{K}_{p, q_{1}}^{\mu, \lambda}\left(R^{n}\right)} \sup _{k_{0} \in Z} 2^{-k_{0} \lambda}\left\{\sum_{k=-\infty}^{k_{0}} 2^{k \lambda p}\right\}^{\frac{1}{p}} \\
& \times \int_{0}^{\infty} \frac{|\Phi(t)|}{t^{1+\gamma}} t^{\frac{n}{q_{1}}} \max \left\{1, t^{-\beta}\right\} t^{\mu-\lambda} d t \\
\leq & C\|b\|_{\dot{\Lambda}_{\beta}\left(R^{n}\right)}\|f\|_{M \dot{K}_{p, q_{1}}^{\mu, \lambda}\left(R^{n}\right)} \int_{0}^{\infty} \frac{|\Phi(t)|}{t} t^{\alpha+\frac{n}{q_{2}}-\lambda} \max \left\{1, t^{\beta}\right\} d t .
\end{aligned}
$$

Similarly,

$$
\begin{aligned}
K_{2} & \leq C\|b\|_{\dot{\Lambda}_{\beta}\left(R^{n}\right)}\|f\|_{M \dot{K}_{p, q_{1}}^{\mu, \lambda}\left(R^{n}\right)} \sup _{k_{0} \in Z} 2^{-k_{0} \lambda}\left\{\sum_{k=-\infty}^{k_{0}} 2^{k \lambda p}\left(\int_{0}^{\infty} \widetilde{\Phi}(t) 2^{(m-1)(\lambda-\mu)} d t\right)^{p}\right\}^{\frac{1}{p}} \\
& \leq C\|b\|_{\dot{\Lambda}_{\beta}\left(R^{n}\right)}\|f\|_{M \dot{K}_{p, q_{1}}^{\mu, \lambda}\left(R^{n}\right)} \int_{0}^{\infty} \frac{|\Phi(t)|}{t^{1+\gamma}} t^{\frac{n}{q_{1}}} \max \left\{1, t^{-\beta}\right\} t^{\mu-\lambda} d t \\
& =C\|b\|_{\dot{\Lambda}_{\beta}\left(R^{n}\right)}\|f\|_{M \dot{K}_{p, q_{1}}^{\mu, \lambda}\left(R^{n}\right)} \int_{0}^{\infty} \frac{|\Phi(t)|}{t} t^{\alpha+\frac{n}{q_{2}}-\lambda} \max \left\{1, t^{\beta}\right\} d t .
\end{aligned}
$$

Now, we consider the case $1<p<\infty$. By Minkowski's inequality, we write

$$
\begin{aligned}
\left\|H_{\Phi, \gamma}^{b} f\right\|_{M \dot{K}_{p, q_{2}}^{\alpha, \lambda}\left(R^{n}\right)} \leq & C\|b\|_{\dot{\Lambda}_{\beta}\left(R^{n}\right)} \sup _{k_{0} \in Z} 2^{-k_{0} \lambda} \int_{0}^{\infty} \widetilde{\Phi}(t)\left\{\sum_{k=-\infty}^{k_{0}} 2^{k \mu p}\left\|f \chi_{C_{k+m-1}}\right\|_{L^{q_{1}\left(R^{n}\right)}}^{p}\right\}^{\frac{1}{p}} d t \\
& +C\|b\|_{\dot{\Lambda}_{\beta}\left(R^{n}\right)} \sup _{k_{0} \in Z} 2^{-k_{0} \lambda} \int_{0}^{\infty} \widetilde{\Phi}(t)\left\{\sum_{k=-\infty}^{k_{0}} 2^{k \mu p}\left\|f \chi_{C_{k+m}}\right\|_{L^{q_{1}\left(R^{n}\right)}}^{p}\right\}^{\frac{1}{p}} d t \\
= & L_{1}+L_{2} .
\end{aligned}
$$

Here, we estimate $L_{1}$ as

$$
\begin{aligned}
L_{1} & \leq C\|b\|_{\dot{\Lambda}_{\beta}\left(R^{n}\right)} \int_{0}^{\infty} \widetilde{\Phi}(t) \sup _{k_{0} \in Z} 2^{-\left(k_{0}+m-1\right) \lambda}\left\{\sum_{k=-\infty}^{k_{0}+m-1} 2^{k \mu p}\left\|f \chi_{C_{k}}\right\|_{L^{q_{1}\left(R^{n}\right)}}^{p}\right\}^{\frac{1}{p}} 2^{(m-1)(\lambda-\mu)} d t \\
& \leq C\|b\|_{\dot{\Lambda}_{\beta}\left(R^{n}\right)}\|f\|_{M \dot{K}_{p, q_{1}}^{\mu, \lambda}\left(R^{n}\right)} \int_{0}^{\infty} \frac{|\Phi(t)|}{t^{1+\gamma}} t^{\frac{n}{q_{1}}} \max \left\{1, t^{-\beta}\right\} t^{\mu-\lambda} d t \\
& =C\|b\|_{\dot{\Lambda}_{\beta}\left(R^{n}\right)}\|f\|_{M \dot{K}_{p, q_{1}}^{\mu, \lambda}\left(R^{n}\right)} \int_{0}^{\infty} \frac{|\Phi(t)|}{t} t^{\alpha+\frac{n}{q_{2}}-\lambda} \max \left\{1, t^{\beta}\right\} d t .
\end{aligned}
$$


Similarly,

$$
\begin{aligned}
L_{2} & \leq C\|b\|_{\dot{\Lambda}_{\beta}\left(R^{n}\right)}\|f\|_{M \dot{K}_{p, q_{1}}^{\mu, \lambda}\left(R^{n}\right)} \int_{0}^{\infty} \frac{|\Phi(t)|}{t^{1+\gamma}} t^{\frac{n}{q_{1}}} \max \left\{1, t^{-\beta}\right\} t^{\mu-\lambda} d t \\
& =C\|b\|_{\dot{\Lambda}_{\beta}\left(R^{n}\right)}\|f\|_{M \dot{K}_{p, q_{1}}^{\mu, \lambda}\left(R^{n}\right)} \int_{0}^{\infty} \frac{|\Phi(t)|}{t} t^{\alpha+\frac{n}{q_{2}}-\lambda} \max \left\{1, t^{\beta}\right\} d t .
\end{aligned}
$$

Thus, we finish the proof of Theorem 3.1.

Now, we deduce the Lipschitz estimates for the commutators of $n$-dimensional fractional Hardy operators on the Morrey-Herz space as a special case of Theorem 3.1.

Corollary 3.5 If $\alpha+\beta+\gamma<\frac{n}{q_{2}^{\prime}}+\lambda$, then under the same conditions as in Theorem 3.1, the commutator of the n-dimensional fractional Hardy operator [15],

$$
H_{\gamma, b} f(x)=\frac{1}{|x|^{n+\gamma}} \int_{|y|<|x|}(b(x)-b(y)) f(y) d y
$$

is bounded from $M \dot{K}_{p, q_{1}}^{\mu, \lambda}\left(R^{n}\right)$ to $M \dot{K}_{p, q_{2}}^{\alpha, \lambda}\left(R^{n}\right)$.

Proof In the operator $H_{\Phi, \gamma}^{b} f(x)$, we replace

$$
\Phi(t)=\Phi_{1}(t)=t^{-n+\gamma} \chi_{(1, \infty)}(t)
$$

then we obtain the commutator of the $n$-dimensional fractional Hardy operator,

$$
H_{\Phi_{1}, \gamma}^{b} f(x)=H_{\gamma, b} f(x)
$$

Hence, by Theorem 3.1

$$
\begin{aligned}
& \left\|H_{\gamma, b} f\right\|_{M \dot{K}_{p, q_{2}}^{\alpha, \lambda}\left(R^{n}\right)} \leq C\|b\|_{\dot{\Lambda}_{\beta}\left(R^{n}\right)}\|f\|_{M \dot{K}_{p, q_{1}}^{\mu, \lambda}\left(R^{n}\right)} \int_{1}^{\infty} t^{\alpha+\beta+\gamma-\frac{n}{q_{2}^{\prime}}-\lambda-1} d t \\
& \leq C\|b\|_{\dot{\Lambda}_{\beta}\left(R^{n}\right)}\|f\|_{M \dot{K}_{p, q_{1}}^{\mu, \lambda}\left(R^{n}\right)^{*}}
\end{aligned}
$$

Thus, the corollary is proved.

Corollary 3.6 If $\alpha+\frac{n}{q_{2}}>\lambda$, then under the same conditions as in Theorem 3.1, the commutator of the adjoint fractional Hardy operator [15],

$$
H_{\gamma, b}^{*} f(x)=\int_{|y| \geq|x|} \frac{1}{|y|^{n-\gamma}}(b(x)-b(y)) f(y) d y,
$$

is bounded from $M \dot{K}_{p, q_{1}}^{\mu, \lambda}\left(R^{n}\right)$ to $M \dot{K}_{p, q_{2}}^{\alpha, \lambda}\left(R^{n}\right)$.

Proof In the operator $H_{\Phi, \gamma}^{b} f(x)$, we replace

$$
\Phi(t)=\Phi_{2}(t)=\chi_{(0,1]}(t)
$$


then we obtain the commutator of the $n$-dimensional adjoint Hardy operator

$$
H_{\Phi_{2}, \gamma}^{b} f(x)=H_{\gamma, b}^{*} f(x)
$$

Thus, by Theorem 3.1

$$
\begin{aligned}
\left\|H_{\gamma, b}^{*} f\right\|_{M \dot{K}_{p, q_{2}}^{\alpha, \lambda}\left(R^{n}\right)} & \leq C\|b\|_{\dot{\Lambda}_{\beta}\left(R^{n}\right)}\|f\|_{M \dot{K}_{p, q_{1}}^{\mu, \lambda}\left(R^{n}\right)} \int_{0}^{1} t^{\alpha+\frac{n}{q_{2}}-\lambda-1} d t . \\
& \leq C\|b\|_{\dot{\Lambda}_{\beta}\left(R^{n}\right)}\|f\|_{M \dot{K}_{p, q_{1}}^{\mu, \lambda}\left(R^{n}\right)} .
\end{aligned}
$$

With this we finish the proof of Corollary 3.6.

\section{Competing interests}

The authors declare that they have no competing interests.

\section{Authors' contributions}

All authors read and approved the final manuscript.

\section{Acknowledgements}

The authors are grateful to the referees for their valuable suggestions and comments, which improved the earlier version of the manuscript.

Received: 1 August 2013 Accepted: 12 December 2013 Published: 30 Dec 2013

\section{References}

1. Liflyand, E, Mórecz, F: The Hausdorff operator is bounded on the real Hardy space $H^{1}(R)$. Proc. Am. Math. Soc. 128 1391-1396 (2000)

2. Kanjin, Y: The Hausdorff operators on real Hardy spaces $H^{P}(R)$. Stud. Math. 148, 37-45 (2001)

3. Liflyand, E, Miyachi, A: Boundedness of the Hausdorff operators in $H^{p}$ spaces, $0<p<1$. Stud. Math. 194, 279-292 (2009)

4. Lin, X, Sun, L: Some estimates on the Hausdorff operator. Acta Sci. Math. 78, 669-681 (2012)

5. Liflyand, E, Mórecz, F: The multi-parameter Hausdorff operators is bounded on the product Hardy space $H^{1}(R \times R)$. Analysis 21, 107-118 (2001)

6. Chen, JC, Fan, DS, Li, J: Hausdorff operators on function spaces. Chin. Ann. Math., Ser. B 33, $537-556$ (2012)

7. Xiao, J: $L^{p}$ and BMO bounds of weighted Hardy-Littlewood averages. J. Math. Anal. Appl. 262,660-666 (2001)

8. Rim, KS, Lee, J: Estimates of weighted Hardy-Littlewood averages on the $p$-adic vactor spa. J. Math. Anal. Appl. 324, 1470-1477 (2006)

9. Chuang, NM, Duang, DV: Weighted Hardy Littlewood operators and commutators on $p$-adic functional spaces. P-Adic Numb. Ultr. Anal. Appl. 5, 65-82 (2013)

10. Wu, QY, Fu, ZW: Sharp estimates of $m$-linear $p$-adic Hardy and Hardy Littlewood Pólya operators. J. Appl. Math. 2011, Article ID 472176 (2011)

11. Fu, ZW, Grafakos, L, Lu, SZ, Zhao, FY: Sharp bounds for $m$-linear Hardy and Hilbert operators. Houst. J. Math. 38 , 225-244 (2012)

12. Coifman, R, Rochberg, R, Weiss, G: Factorization theorems for Hardy spaces in several variables. Ann. Math. 103 611-635 (1976)

13. Gao, G, Jia, H: Boundedness of commutators of high dimensional Hausdorff operator. J. Funct. Spaces Appl. 2012 Article ID 54120 (2012)

14. Tang, CQ, Xue, F, Yu, Z: Commutators of weighted Hardy operators on Herz-type spaces. Ann. Pol. Math. 101, 267-273 (2011)

15. Fu, ZW, Liu, ZG, Lu, SZ, Wang, HB: Characterization for commutators of n-dimensional fractional Hardy operators. Sci. China Ser. A 50, 1418-1426 (2007)

16. Lu, SZ: Yang, DC, Hu, GE: Herz Type Spaces and Their Applications. Science Press, Beijing (1995)

17. Lu, SZ, Yang, DC, Zhou, ZS: Sublinear operators with rough kernel on generalized Morrey spaces. Hokkaido Math. J. 27, 219-232 (1998)

18. Paluszynski, M: Characterization of Bosev spaces via the commutator operator of Coifman, Rochberg and Weiss. Indiana Univ. Math. J. 44, 1-18 (1995)

19. Chen, JC, Fan, DS, Zhang, CJ: Multilinear Hausdorff operators and their best constants. Acta Math. Sin. Engl. Ser. 28 1521-1530 (2012) 